\title{
fMRI goes individual
}

Functional magnetic resonance data are traditionally analyzed on a population level, but new work shows that meaningful information can be extracted from individual subjects.

Functional magnetic resonance imaging (fMRI) visualizes the blood oxygen level-dependent (BOLD) signal in the brain, which indexes neural activity. However, this signal is noisy and variable. Therefore, most studies average data from many people. "By far the majority of studies have been conducted and analyzed that way, and it has taught us a lot about which regions are generally involved in which tasks across people," says Emily Finn from Yale University.

Finn, Xilin Shen and their colleagues in the lab of Todd Constable at Yale University decided to analyze fMRI data from individual subjects. Finn explains that they wanted to prove that data from a single subject are actually meaningful and reliable. The researchers used data from 126 subjects that had been acquired over two consecutive days during two rest sessions and four sessions that involved a variety of different tasks.

Traditionally, changes in the magnitude of the BOLD signal are used to analyze brain activity. Finn and her colleagues chose to look at functional connectivity patterns instead. "We were looking at the pattern synchrony between different pairs of regions over the course of the scan," explains Finn. Although not novel, this approach gives the data another dimension. More specifically, the researchers divided the brain into 268 regions or nodes and determined whether these regions exhibited correlated activity over time, resulting in connectivity matrices of 268 by 268 nodes for each session. Having established these connectivity profiles, Finn and her colleagues set out to determine whether $\mathrm{MRI}$ as a technique is robust and reliable enough to capture individual variability across several days and cognitive tasks. In other words, does it matter what people are doing, or does it matter who they are?

To answer this question, the researchers compared a person's connectivity matrix from a particular session with the connectivity matrices from all subjects obtained during a different day. "We wanted to show that for the same person doing two different things, you can still pick someone out of a crowd, regardless of how their brain is engaged during the scan," explains

STRUCTURAL BIOLOGY

\section{STRUCTURE IN THE CELLULAR CONTEXT}

Researchers develop an approach based on solid-state nuclear magnetic resonance (NMR) to study the structure of an intrinsically disordered protein under nearnative conditions.

Traditionally, investigations into the molecular structure of a protein are carried out under purified conditions highly dissimilar to the warm, cozy environment of a cell. Biologists have long recognized the incongruity of studying protein structure under such abnormal conditions, but the dearth of methods for studying protein structure in the cellular context has forced researchers to make do with purified samples.

One promising approach that has enabled structural studies in a more native context is in-cell NMR. However, in studies using this solution NMR-based approach, the proteins of interest have to be analyzed at unnaturally high levels to generate a sufficient NMR signal. In-cell NMR studies have suggested that protein structure is not dramatically affected by the cellular environment; however, with the concentration of the protein of interest in those studies far exceeding endogenous levels, only a small amount of the protein of interest could be expected to be available to interact with other entities in the cell.

Susan Lindquist of the Massachusetts Institute of Technology and her colleagues, including first author Kendra Frederick, who is now running her own lab at UT Southwestern, recently devised an approach to help bridge the gap between the cellular environment and the ability to determine protein structure. They have been studying the yeast prion protein Sup35, which contains an intrinsically disordered region. A prion by definition can exist in different forms-a normal soluble form or a heritable amyloid form-mediated by cellular remodeling factors. Studying the structure of such a protein outside its cellular context would therefore reveal very little about what it does in the cell. 

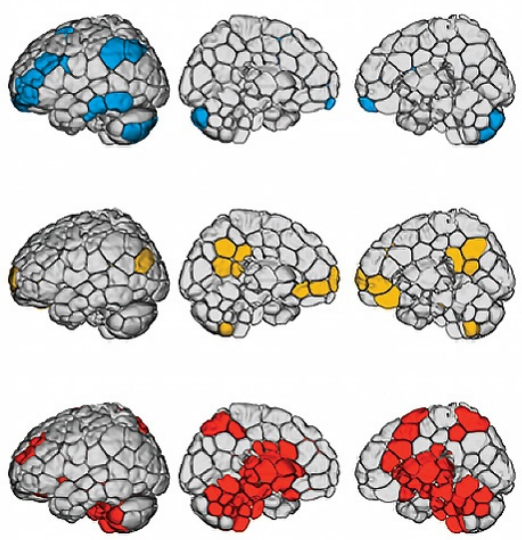

Regions in the brain with correlated activity patterns. Reproduced with permission from Finn et al. (2015).
Finn. They indeed found that it was possible to identify a person from his or her brain activity data even when the data were acquired in different sessions on different days.

An interesting question is whether brain connectivity patterns are similar in genetically related subjects. In fact, many subjects in the study were twins or had other relatives who participated. "We expected that when we got the identity wrong we might have been more likely to confuse people with their twin or their sibling," concedes Finn, but this was not the case. The researchers did not find a strong trend for similar brain activity patterns in relatives.

However, brain connectivity profiles did correlate with an innate form of intelligence called fluid intelligence. Using the connectiv-

ity profiles, Finn and her colleagues could predict to a certain degree how individuals would perform in an intelligence test that assessed pattern recognition and problem-solving skills independently of acquired knowledge.

But Finn is also cautious about the implications. Although the results are statistically significant, such individual fMRI analyses are not ready for practical application in a clinical setting (for example, to guide treatment). Nevertheless, the findings are exciting because they suggest "that these connectivity profiles are more stable than we thought and more reliable than we thought. [They] open the door to doing more on an individual-subject level," says Finn.

Nina Vogt

\section{RESEARCH PAPERS}

Finn, E.S. et al. Functional connectome fingerprinting: identifying individuals using patterns of brain connectivity. Nat. Neurosci. 18, 1664-1671 (2015).

To study the structure of Sup35 in a native-like environment at endogenous levels of the protein, Lindquist, Frederick and colleagues turned to solid-state NMR. The sensitivity of solid-state NMR is very low, but combining the technique with dynamic nuclear polarization-in which electron spin polarization from a polarizing reagent is transferred to nuclei in a microwave-driven reaction-substantially boosts the NMR signals.

The final piece of the puzzle was developing a way to clearly see the Sup35 NMR signals without interference from other cellular entities. The researchers prepared recombinant Sup35 labeled with NMR-active carbon-13 and then added it to an NMRinvisible deuterium-labeled cell lysate, thus ensuring that proton and carbon-13 NMR signals would be detected from only the protein and not the cellular background.

The dynamic nuclear polarization solid-state NMR data indicated that the region of Sup 35 that is highly disordered in the purified protein took on a $\beta$-sheet structure in lysates. Sup35 is known to interact directly with chaperone proteins; the researchers thus concluded that such interactions have an important role in shaping the protein's structure in its native environment.

Sup35 is certainly not unique in having a structure that is sensitive to the cellular environment; more than $30 \%$ of the proteome is predicted to have at least some intrinsic disorder. Moreover, protein misfolding has a key role in multiple human diseases, including devastating conditions such as Alzheimer's, Parkinson's and Huntington's diseases. Lindquist, Frederick and colleagues' method to study protein structure in the native context therefore may also have a key role in helping scientists design new and more effective therapies.

Allison Doerr

RESEARCH PAPERS

Frederick, K.K. et al. Sensitivity-enhanced NMR reveals alterations in protein structure by cellular milieus. Cell 163, 620-628 (2015). 